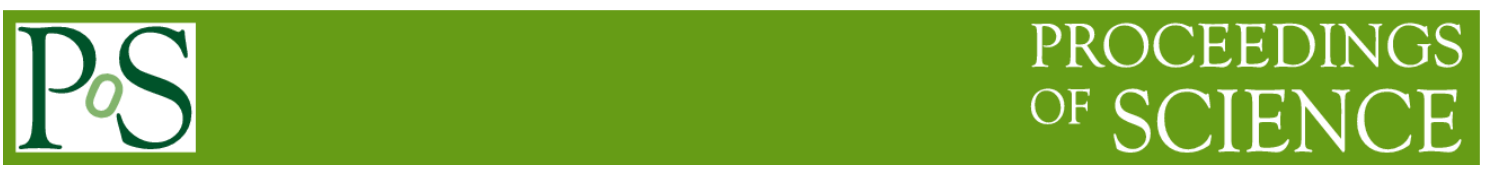

\title{
Radiation study of the target station for the EMuS baseline scheme
}

\section{Guang Zhao'}

Institute of High Energy Physics

19B Yuquan Road, Beijing, China

E-mail:zhaog@ihep.ac.cn

In this presentation, a radiation study of the target station for the EMuS baseline scheme is reported. A detailed study for the $5-\mathrm{kW}$ proton beam is firstly reported and a preliminary study for the $25-\mathrm{kW}$ proton beam is also presented.

The 21st international workshop on neutrinos from accelerators (NuFact2019) August 26 - August 31, 2019

Daegu, Korea

\section{${ }^{1}$ Speaker}




\section{Introduction}

The Experimental Muon Source (EMuS) is a proposed facility at China Spallation Neutron Source (CSNS) [1]. EMuS, which is an additional platform to CSNS, aims to provide muon beams for different applications such as neutrino physics, muSR, etc. The baseline design uses up to $25 \mathrm{~kW}$ proton beam. The target station of EMuS is consisting of a long carbon target in conical shape, a superconducting capture solenoids system that produce adiabatic magnetic field and shields inside the solenoids to protect them from radiation (Fig. 1). The target is situated in the superconducting solenoids and secondary particles are collected forwardly. As a result, high radiation is produced in the target station. In this presentation, we will present the radiation study for the baseline scheme.

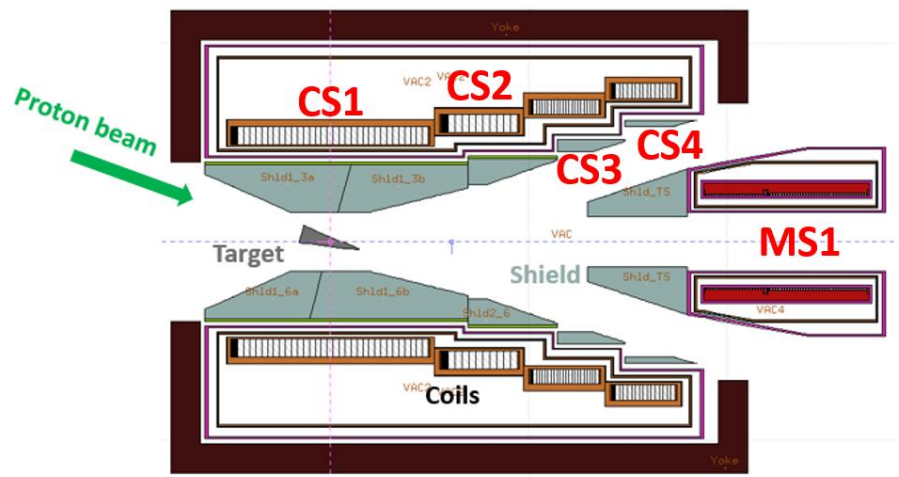

Figure 1: EMuS target station

\section{Radiation study for the $5-\mathrm{kW}$ beam}

We firstly report the study for the $5 \mathrm{~kW}$ proton beam. The most radiation vulnerable material is the epoxy which is used as the insulator and the material to bond the cables. It can tolerate a maximum of $7 \mathrm{MGy}$ dose before it experiences a $10 \%$ degradation in its shear modulus [2]. We optimize the tungsten shield position, thickness and materials (Fig. 2). Results show that after the optimization, the peak doses on all the cables satisfy this 7 MGy limit for a 10 year operation (Fig. $3)$.
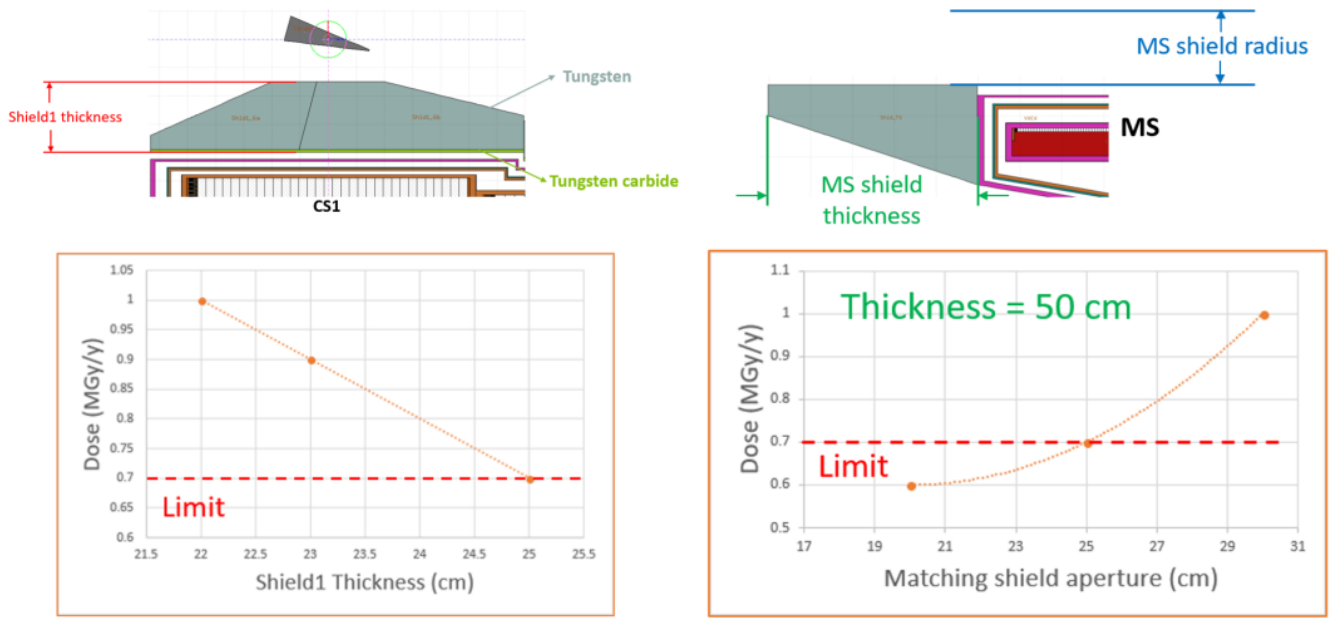

Figure 2: Shielding optimization for CS1 (left) and MS1 (right). 


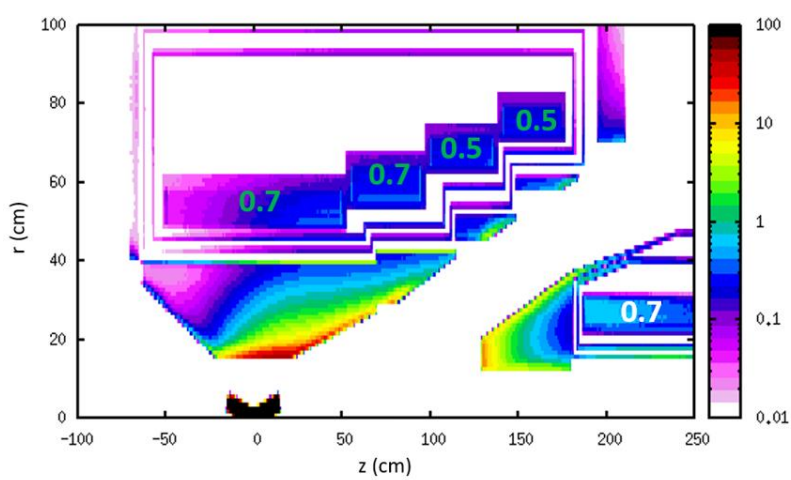

Figure 3: Peak dose distributions. The numbers (in units of MGy/year) in the plot indicate the peak dose value on the coils.

Another radiation effect we should consider is the neutron fluence on the superconducting solenoids. The electrical conductivity of the stabilizer of the solenoids degrade in high-flux neutron environment, which can lead to instability of the superconducting system [3, 4]. Our calculation shows that the aluminum stabilized cable can stand for a 5-month continuous run after it needs to be warmed up to room temperature to get the full recovery (Fig. 4).

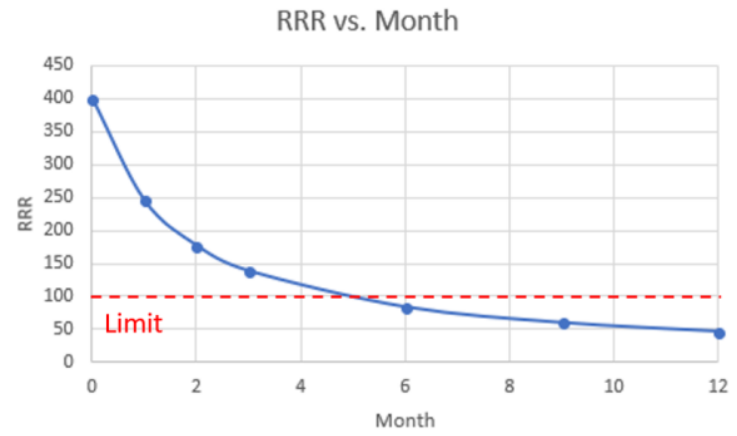

Figure 4: Residual Resistivity Ratio (RRR) for the stabilizers over operation time.

We have also performed the thermal analysis for superconducting solenoids considering the helium cooling and the aluminum 1100 thermal bridge. Results show the superconducting solenoid system can safely run for 5 months (Fig. 5).

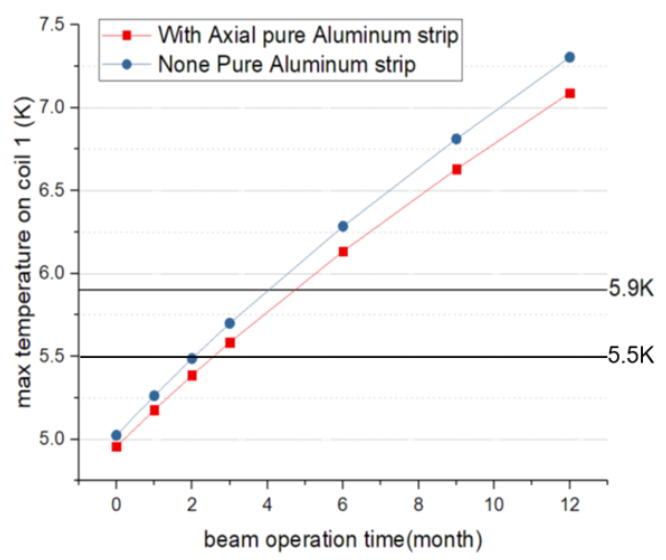

Figure 5: Temperature on the coils over operation time. 
The simulation results show a reliable design for the target station with $5-\mathrm{kW}$ proton beam regarding the radiation.

\section{Radiation study for the $25-\mathrm{kW}$ beam}

As CSNS is planned to upgrade to phase-II [5] and EMuS will use $25 \mathrm{~kW}$ of the CSNS proton beam, the radiation study for the $25-\mathrm{kW}$ beam is ongoing.

For the $25 \mathrm{~kW}$ beam, radiation level will be much higher. The layout of the superconducting solenoids should be changed, and more shields need to be equipped. Our early study show a hybrid-shield-design can satisfy the requirement for the dose on the solenoids.

More studies will be carried out in the future for the $25-\mathrm{kW}$ proton beam case.

\section{References}

[1] Hesheng Chen and Xun-Li Wang, China's first pulsed neutron source, Nature Materials 15, 689691 (2016).

[2] A. Zeller et al, Radiation Hard Coils, http://supercon.lbl.gov/WAAM.

[3] J. Horak and T. Blewitt, Isochronal Recovery of Fast Neutron Irradiated Metals, J. Nucl. Mat., 49 (1973/74) 161-180.

[4] M. Guian et al, Defect Production and Recovery in FCC Metals Irradiated at 4.2 K, J. Nucl. Mater. $133 \& 134$ (1985) 357-360.

[5] J. Wei et al, Nucl. Instr. and Meth in Phys. Research A 600 (2009) 10-13. 